\title{
Mutation of IDH1 aggravates the fatty acid-induced oxidative stress in HCT116 cells by affecting the mitochondrial respiratory chain
}

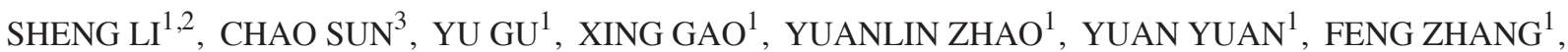 \\ PEIZHEN HU ${ }^{1}$, WEIHUA LIANG ${ }^{1}$, KAIYU CAO ${ }^{1}$, JIN ZHANG $^{1}$, ZHE WANG $^{1}$ and JING YE ${ }^{1}$ \\ ${ }^{1}$ State Key Laboratory of Cancer Biology and Department of Pathology, Xijing Hospital, \\ The Fourth Military Medical University, Xi'an, Shaanxi 710032; ${ }^{2}$ Department of Pathology and \\ School of Basic Medical Sciences, Xi'an Medical University, Xi'an, Shaanxi 710021; \\ ${ }^{3}$ Department of Neurology, Tangdu Hospital, The Fourth Military Medical University, \\ Xi'an, Shaanxi 710038, P.R. China
}

Received February 15, 2018; Accepted October 12, 2018

DOI: $10.3892 / \mathrm{mmr} .2019 .9903$

\begin{abstract}
Increasing evidence has indicated that mutations of isocitrate dehydrogenase $1 / 2$ (IDH1/2) contribute to the metabolic reprogramming of cancer cells; however their functions in lipid metabolism remain unknown. In the present study, the parental and IDH1 (R132H/+) mutant HCT116 cells were treated with various concentrations of oleic acid (OA) or palmitic acid (PA) in the presence or absence of glucose. The results demonstrated that mutation of IDH1 exacerbated the effects of OA and PA on cell viability and apoptosis, and consistently elevated the production of reactive oxygen species in HCT116 cells, particularly in the absence of glucose. Furthermore, mutation of IDH1 inhibited the rate of fatty acid oxidation (FAO), but elevated the glucose consumption in HCT116 cells. The results of immunoblotting and reverse transcription-quantitative polymerase chain reaction (RT-qPCR) indicated that the expression of glucose transporter 1 was upregulated, whereas that of carnitine palmitoyl transferase 1 was downregulated in IDH1 mutant HCT116 cells. Although mitochondrial DNA quantification demonstrated that mutation of IDH1 had no effect on the quantity of mitochondria, immunoblotting and RT-qPCR revealed that mutation of IDH1 in HCT116 cells significantly downregulated the expression of cytochrome $c$ (CYCS) and CYCS oxidase IV, two important components in mitochondrial respiratory chain. These
\end{abstract}

Correspondence to: Professor Jing Ye or Professor Zhe Wang, State Key Laboratory of Cancer Biology and Department of Pathology, Xijing Hospital, The Fourth Military Medical University, 169 Changle West Road, Xi'an, Shaanxi 710032, P.R. China

E-mail: yejing@fmmu.edu.cn

E-mail: zhwang@fmmu.edu.cn

Key words: isocitrate dehydrogenase mutation, fatty acid oxidation, glycolysis, oxidative stress, respiratory chain results indicated that mutation of IDH1 aggravated the fatty acid-induced oxidative stress in HCT116 cells, by suppressing FAO and disrupting the mitochondrial respiratory chain. The results of the present study may provide novel insight into therapeutic strategies for the treatment of cancer types with IDH mutation.

\section{Introduction}

In addition to genetic and epigenetic alterations, metabolic reprogramming has been identified as a hallmark of numerous types of malignant tumour, as it is associated with cellular transformation and cancer progression (1). Apart from altered glucose metabolism, a recent study demonstrated that cancer cells exhibit aberrant lipid metabolism to facilitate cell growth, proliferation, differentiation and motility (2). As the major components of triglycerides (TGs), fatty acids (FA) serve essential roles in the synthesis of structural membranes, signalling pathways and energy homeostasis (3). In energy metabolism, fatty acid oxidation (FAO) also provides an important source of reducing equivalents and ATP, which assists the survival of cancer cells (4); however, FAO increases the production of reactive oxygen species (ROS) via the mitochondria (5). ROS able to modify biological macromolecules, including DNA, lipids and proteins (5). Additionally, the levels of ROS are upregulated in cancer cells (6).

Recurrent mutations in isocitrate dehydrogenase $1 / 2$ (IDH1/2) have been identified in gliomas, acute myeloid leukemia (AML) and chondrosarcomas (7-10). Wild type IDH1/2 catalyses the conversion of isocitrate to $\alpha$-ketoglutarate $(\alpha-K G)$ via the reduction of nicotinamide-adenine dinucleotide phosphate $(\mathrm{NADP})^{+}(11)$, and serves important roles in the regulation of redox status, lipogenesis, glucose and amino acid metabolism (12); however, it is well established that oncogenic IDH mutations promote the NADPH-dependent reduction of $\alpha$-KG into 2-hydroxyglutarate (2-HG) (13). As an oncometabolite, $2-\mathrm{HG}$ is a competitive inhibitor of $\alpha-\mathrm{KG}$-dependent dioxygenases, including histone demethylases and 
5-methylcytosine hydroxylases, which can result in complex genetic and epigenetic alterations (14). The roles of wild type IDH1 in lipid biosynthesis of liver and adipose tissues have been established $(15,16)$ and its roles in tumorigenesis have also been investigated (17). A recent study has demonstrated that D-2-HG-induced hypersuccinylation contributes to the tumorigenicity of cells with IDH mutations, consequently resulting in mitochondrial dysfunction (18); however the regulatory functions of mutant IDH in lipid metabolism remain unknown.

In the present study, the effects of FAs on the growth and apoptosis of human colon carcinoma HCT116 cells with mutant IDH1 were investigated. Furthermore, ROS production and mitochondrial dysfunction were determined to identify the roles of mutant IDH1 in FA metabolism. The findings of the present study are not only important to understand the functions of mutant IDH in tumour metabolic reprogramming, but also provide novel insight into the therapeutic strategies for patients with cancer and mutant IDH.

\section{Materials and methods}

Cell culture. The parental and IDH1 (R132H/+) HCT116 cells (Horizon Discovery, Cambridge, UK) were cultured in McCoy's 5A medium (cat. no. 16600082, Gibco; Thermo Fisher Scientific, Inc., Waltham, MA, USA) with $10 \%$ fetal bovine serum (FBS; cat. no. 10099141, Gibco; Thermo Fisher Scientific, Inc.) at $37^{\circ} \mathrm{C}$ in an atmosphere containing $5 \% \mathrm{CO}_{2}$. The heterozygous IDH1 (R132H/+) HCT116 cells were generated from the parental cells that were transfected with the IDH1 (R132H) allele, which resulted in >100-fold upregulation of D-2-HG compared with the parental cells (19). Cells $\left(5 \times 10^{6}\right)$ were treated with $0,50,100,200$ and $400 \mu \mathrm{M}$ oleic acid (OA) or palmitic acid (PA) (Sigma-Aldrich; Merck KGaA, Darmstadt, Germany) in the presence or absence of $5.5 \mathrm{mM}$ glucose at $37^{\circ} \mathrm{C}$ for $24 \mathrm{~h}$.

PA and OA were prepared with a bovine serum albumin (BSA) (Sigma-Aldrich; Merck KGaA) conjugate. Briefly, $0.25 \mathrm{ml} 200 \mathrm{mM}$ PA or OA solutions (in anhydrous ethanol) were added into $10 \mathrm{ml}$ FA-free BSA (10\%, A4612, Sigma-Aldrich; Merck KGaA) in PBS, and mixed gently for $\geq 2$ h until completely dissolved. Control BSA was prepared by adding the same amount of ethanol into $10 \%$ BSA solution. BSA-FA conjugates were further diluted in medium to reach a final concentration of $0,50,100,200$ or $400 \mu \mathrm{M}$. All solutions were aliquoted and frozen at $-80^{\circ} \mathrm{C}$.

MTT assay. The number of viable cells was determined using an MTT assay. The cells were plated in a 96-well culture plate. Following treatment with $0,50,100,200$ or $400 \mu \mathrm{M}$ $\mathrm{OA}$ or $\mathrm{PA}$, in the presence or absence of $5.5 \mathrm{mM}$ glucose, at $37^{\circ} \mathrm{C}$ for $24 \mathrm{~h}$, MTT solution was added at a final concentration of $0.5 \mathrm{mg} / \mathrm{ml}$. Following $1 \mathrm{~h}$ of incubation at $37^{\circ} \mathrm{C}$ in the dark, the media were removed and purple formazan was solubilized using dimethyl sulfoxide (Sigma-Aldrich; Merck KGaA). The solutions were collected and transferred to a 96-well plate; the absorbance was measured at a wavelength of $540 \mathrm{~nm}$ using a microplate reader (Thermo Fisher Scientific, Inc.).
Propidium iodide (PI) staining. Parental and IDH1 (R132H/+) HCT116 cells were seeded at a density of $1 \times 10^{5}$ cells/well on coverslips in 12-well plates and cultured in McCoy's 5A medium overnight. Treatment with $0,50,100,200$ or $400 \mu \mathrm{M}$ $\mathrm{OA}$ or $\mathrm{PA}$, in the presence or absence of $5.5 \mathrm{mM}$ glucose, was performed at $37^{\circ} \mathrm{C}$ for $24 \mathrm{~h}$. Medium was removed from the plates and the cells were rinsed cells twice in PBS. Staining solution containing PI (cat. no. P3566; Invitrogen; Thermo Fisher Scientific, Inc.) was added at room temperature for 5 min. Cells were viewer using a fluorescence microscope (U-RFL-T; Olympus Corporation, Tokyo, Japan) under x1,000 magnification. The numbers of positive cells across six fields of view were counted and averaged.

MitoTracker staining. Parental and IDH1 (R132H/+) HCT116 cells were seeded at a density of $4 \times 10^{5}$ cells/well on coverslips in 12-well plates and cultured in McCoy's 5A medium overnight. The medium was removed from the plates and prewarmed $\left(37^{\circ} \mathrm{C}\right)$ staining solution containing Mito Tracker Red CMXRos probe (cat. no. M7512, Invitrogen; Thermo Fisher Scientific, Inc.) was added at $37^{\circ} \mathrm{C}$ for $20 \mathrm{~min}$. The staining solution was replaced with fresh prewarmed PBS. The PBS covering the cells was subsequently removed and replaced with $4 \%$ formaldehyde at $37^{\circ} \mathrm{C}$ for $15 \mathrm{~min}$. Following fixation, the cells were rinsed a number of times in buffer. Nuclei were stained with Hoechst at room temperature for $2 \mathrm{~min}$. Cells were viewed using a fluorescence microscope (U-RFL-T; Olympus Corporation) under x1,000 magnification.

Dichloro-dihydro-fluorescein diacetate (DCFH-DA) staining. Parental and IDH1 (R132H/+) HCT116 cells were seeded at a density of $1 \times 10^{5}$ cells/well on coverslips in 12-well plates and cultured in McCoy's 5A medium overnight. Cells were treated with $400 \mu \mathrm{M}$ OA or PA in the presence or absence of $5.5 \mathrm{mM}$ glucose at $37^{\circ} \mathrm{C}$ for $24 \mathrm{~h}$. The medium was removed from the plates and staining solution containing DCFH-DA (cat. no. S0033; Beyotime Institute of Biotechnology, Haimen, China) was added at $37^{\circ} \mathrm{C}$ for $20 \mathrm{~min}$. The cells were rinsed twice in serum-free medium. The medium was replaced with $4 \%$ formaldehyde at $37^{\circ} \mathrm{C}$ for $15 \mathrm{~min}$. Following fixation, the cells were rinsed twice. The nuclei were stained with Hoechst at room temperature for $2 \mathrm{~min}$. Cells were viewed using a fluorescence microscope (U-RFL-T; Olympus Corporation) under x1,000 magnification, followed by quantitative analysis using flow cytometry (FlowJo v.7.6.3; FlowJo, LLC, Ashland, OR, USA).

Bodipy 493/503 staining. Parental and IDH1 (R132H/+) HCT116 cells were seeded at a density of $1 \times 10^{5}$ cells/well on coverslips in 12-well plates and cultured in McCoy's 5A medium overnight. The cells were treated with $400 \mu \mathrm{M}$ OA or PA in the presence or absence of $5.5 \mathrm{mM}$ glucose at $37^{\circ} \mathrm{C}$ for $24 \mathrm{~h}$. The cells were rinsed twice in PBS. The cells were fixed with $4 \%$ formaldehyde at $37^{\circ} \mathrm{C}$ for $15 \mathrm{~min}$. Subsequently, the cells were rinsed twice in PBS. The PBS was removed and staining solution containing Bodipy 493/503 (Invitrogen; Thermo Fisher Scientific, Inc.) was added at room temperature for $5 \mathrm{~min}$. The cells were rinsed twice. The nuclei were stained with Hoechst at room temperature for $2 \mathrm{~min}$. The cells were viewed using a fluorescence microscope (U-RFL-T; Olympus 
Corporation) under x1,000 magnification, followed by quantitative analysis using flow cytometry (FlowJo v.7.6.3; FlowJo, LLC).

Determination of intracellular TG levels. The cells in each group were homogenized in lysis buffer. A volume of $200 \mu \mathrm{l}$ cell lysate was added to $800 \mu \mathrm{l}$ methanol/chloroform $(2: 1 \mathrm{v} / \mathrm{v})$. After vigorously mixing several times, the tubes were centrifuged at 3,000 x g for $5 \mathrm{~min}$. The lower phase containing lipids was removed and placed in vials, and dried under nitrogen steam. The lipids were resolved in $200 \mu 12 \%$ Triton X-100 and the levels of intracellular TG in the cells were analyzed using a TG test kit (Wako Pure Chemical Industries, Ltd., Osaka, Japan).

Determination of malondialdehyde (MDA) and 4-hydroxy-2-nonenal (4-HNE). The cellular levels of MDA and 4-HNE were determined using a microscale MDA assay kit (cat. no. A003, Nanjing Jiancheng Bioengineering Institute Co., Ltd., Nanjing, China) and a 4-HNE adduct ELISA kit (cat. no. STA-838, Cell Biolabs, Inc., San Diego, CA, USA) according to the manufacturer's protocols.

Determination of FAO. Parental and IDH1 (R132H/+) HCT116 cells were cultured at $37^{\circ} \mathrm{C}$ for $12 \mathrm{~h}$ in McCoy's 5A medium containing $10 \%$ FBS. Cells $\left(2 \times 10^{6}\right)$ were then washed twice with Hanks' balanced salt solution [HBSS; $137.93 \mathrm{mM}$ $\mathrm{NaCl}, 5.33 \mathrm{mM} \mathrm{KCl}, 4.17 \mathrm{mM} \mathrm{NaHCO} 3,0.441 \mathrm{mM} \mathrm{KH}_{2} \mathrm{PO}_{4}$, $0.338 \mathrm{mM} \mathrm{Na}_{2} \mathrm{HPO}_{4}, 5.56 \mathrm{mM}$ glucose, $0.407 \mathrm{mM} \mathrm{MgSO}_{4}$, $0.493 \mathrm{mM} \mathrm{MgCl}_{2}$ and $1.26 \mathrm{mM} \mathrm{CaCl}_{2}$ (Beyotime Institute of Biotechnology)]. The experiments were performed in triplicate on a 12-well plate. Briefly, $500 \mu \mathrm{l}$ HBSS containing $22 \mu \mathrm{M}{ }^{3} \mathrm{H}$-labelled OA or PA $(1 \mu \mathrm{Ci} /$ well) (GE Healthcare Life Sciences, Little Chalfont, UK) with $0.5 \mathrm{mg} / \mathrm{ml}$ FA-free BSA was added into each well. Following incubation for 4 and $8 \mathrm{~h}$ at $37^{\circ} \mathrm{C}$, the medium was collected and transferred into a glass tube, followed by extraction with $8 \mathrm{ml}$ methanol/chloroform $(2: 1, \mathrm{v} / \mathrm{v})$ and $0.5 \mathrm{ml} 2 \mathrm{M} \mathrm{KCl} / 2 \mathrm{M} \mathrm{HCl}$. The aqueous phase containing ${ }^{3} \mathrm{H}_{2} \mathrm{O}$ was transferred into a fresh tube and further extracted using methanol/chloroform (2:1 v/v) and $2 \mathrm{M}$ $\mathrm{KCl} / 2 \mathrm{M} \mathrm{HCl}$ solution. Subsequently, $10 \mathrm{ml}$ scintillation solution (PerkinElmer, Inc., Waltham, MA, USA) was added to the aqueous phase, and radioactivity was measured using a liquid scintillation counter (L6500; Beckman Coulter, Inc., Brea, CA, USA). The rate of FAO was presented as the production of radioactive water or water-soluble metabolites at 4 and $8 \mathrm{~h}$.

Glucose consumption analysis. Parental and IDH1 (R132H/+) HCT116 cells were seeded in 24-well plates, at a density of $1 \times 10^{5}$ cells/well. Following overnight incubation, the cells were cultured in McCoy's 5A medium with $5.5 \mathrm{mM}$ glucose and $400 \mu \mathrm{M} \mathrm{OA}$ or PA at $37^{\circ} \mathrm{C}$ for 4 and $8 \mathrm{~h}$. The glucose levels in the medium were determined using a high sensitivity glucose assay kit (Sigma-Aldrich; Merck KGaA) according to the manufacturer's protocols.

Western blot analysis. The cells in each group were homogenized in lysis buffer [20 mM Tris ( $\mathrm{pH} 7.5), 150 \mathrm{mM} \mathrm{NaCl}$, $1 \%$ Triton X-100, sodium pyrophosphate, $\beta$-glycerophosphate, EDTA, $\mathrm{Na}_{3} \mathrm{VO}_{4}$ and leupeptin] (Beyotime Institute of
Biotechnology). The protein concentrations in the soluble lysates were determined by the Bradford method (Bio-Rad Laboratories, Inc., Hercules, CA, USA). Briefly, proteins (10-50 $\mu \mathrm{g} /$ lane) were separated by 10 and $15 \%$ SDS-PAGE and transferred onto a polyvinylidene fluoride membranes (EMD Millipore, Billerica, MA, USA). The membranes were blocked using 5\% skimmed milk at room temperature for $1 \mathrm{~h}$. The membranes were incubated with each primary antibody at $4^{\circ} \mathrm{C}$ overnight, followed by incubation with horseradish peroxidase-conjugated secondary antibodies (cat. nos. NA931 and NA934; 1:5,000; GE Healthcare Life Sciences) at room temperature for $1 \mathrm{~h}$. The bands were visualized using enhanced chemiluminescence substrate (Applygen Technologies, Inc., Beijing, China) and exposed using X-ray film.

The antibodies used for immunoblotting were as follows: IDH1 (R132H; 1:2,000; cat. no. D339-3; Medical \& Biological Laboratories Co., Ltd., Nagoya, Japan); glucose transporter 1 (GLUT1; cat. no.07-1401; 1:10,000; EMD Millipore); carnitine palmitoyl transferase 1 (CPT1; cat. no. CPT1L12-A; 1:5,000; Alpha Diagnostic International, Inc., San Antonio, TX, USA); cytochrome $c$ (CYCS; cat.no. 556433; 1:1,000; BD Pharmingen; BD Biosciences, Franklin Lakes, NJ, USA); CYCS oxidase IV (Cox4; cat. no. YM3033; 1:1,000; ImmunoWay Biotechnology Co., Plano, TX, USA); and $\beta$-tubulin (cat. no. KM9003T; 1:1,000; Tianjin Sungene Biotech Co., Ltd., Tianjin, China).

Reverse transcription-quantitative polymerase chain reaction $(R T-q P C R)$. Total RNA was isolated from cells using TRIzol ${ }^{\circledR}$ (Invitrogen; Thermo Fisher Scientific, Inc.) according to the manufacturer's protocols. A total of $10 \mu \mathrm{g}$ RNA was reverse-transcribed into cDNA using a Prime Script RT Master Mix kit (Takara Biotechnology Co., Ltd., Dalian, China) (conditions: $37^{\circ} \mathrm{C}$ for $15 \mathrm{~min}$, followed by $85^{\circ} \mathrm{C}$ for $5 \mathrm{sec}$ ); qPCR was performed using $\mathrm{SYBR}^{\circledR}$ Premix Ex Taq $^{\mathrm{TM}}$ II (Takara Biotechnology Co., Ltd.) with an ABI Step One plus Real-time PCR system (Applied Biosystems; Thermo Fisher Scientific, Inc.). The thermocycling conditions used for $\mathrm{qPCR}$ were as follows: $95^{\circ} \mathrm{C}$ for $30 \mathrm{sec}$, followed by 40 cycles of $95^{\circ} \mathrm{C}$ for $5 \mathrm{sec}$ and $60^{\circ} \mathrm{C}$ for $30 \mathrm{sec}$. Each analysis was performed in three to six replicates. Primers used for RT-qPCR are presented in Table I. The relative gene expression was normalized to the reference gene $\beta$-actin using the $2^{-\Delta \Delta \mathrm{Cq}}$ method (20).

Mitochondrial DNA quantification. Total DNA was extracted using the Universal Genomic DNA Extraction kit (Takara Biotechnology Co., Ltd.). The mtDNA copy number was determined using qPCR which was performed using $\mathrm{SYBR}^{\circledR}$ Premix Ex Taq ${ }^{\mathrm{TM}}$ II (Takara Biotechnology Co., Ltd.) with an ABI Step One plus Real-time PCR system (Applied Biosystems; Thermo Fisher Scientific, Inc.). Primers of the mtDNA-encoded gene NADH dehydrogenase subunit 1 (ND1) were (forward) 5'-CCCTAAAACCCGCCACATCT-3' and (reverse) 5'-GAG CGATGGTGAGAGCTAAGGT-3', and primers of the nuclear gene hemoglobin subunit $\beta$ (HGB) were (forward) 5'-GAA GAGCCAAGGACAGGTAC-3' and (reverse) 5'-CAACTT CATCCACGTTCACC-3'. The thermocycling conditions used for mtDNA-encoded gene ND1 qPCR were as follows: $50^{\circ} \mathrm{C}$ for $2 \mathrm{~min}, 95^{\circ} \mathrm{C}$ for $2 \mathrm{~min}$, followed by 40 cycles of $95^{\circ} \mathrm{C}$ for $15 \mathrm{sec}$ and $60^{\circ} \mathrm{C}$ for $1 \mathrm{~min}$. The thermocycling conditions used for nuclear gene $\mathrm{HGB}$ qPCR were as follows: $50^{\circ} \mathrm{C}$ for $2 \mathrm{~min}$, 
Table I. Polymerase chain reaction primer sequences (human).

\begin{tabular}{|c|c|c|c|}
\hline Gene & Primers & Sequences $\left(5^{\prime}-3^{\prime}\right)$ & Accession no. ${ }^{\mathrm{a}}$ \\
\hline \multirow[t]{2}{*}{ ACTB } & Forward & CCAACCGCGAGAAGATGA & NM_001101 \\
\hline & Reverse & CCAGAGGCGTACAGGGATAG & \\
\hline \multirow[t]{2}{*}{ GLUT1 } & Forward & CTGGCATCAACGCTGTCTTC & NM_006516 \\
\hline & Reverse & GTTGACGATACCGGAGCCAA & \\
\hline \multirow[t]{2}{*}{ GLUT4 } & Forward & TGGGCGGCATGATTTCCTC & NM_001042 \\
\hline & Reverse & GCCAGGACATTGTTGACCAG & \\
\hline \multirow[t]{2}{*}{ CPT1 } & Forward & TCCAGTTGGCTTATCGTGGTG & NM_001876 \\
\hline & Reverse & TCCAGAGTCCGATTGATTTTTGC & \\
\hline \multirow[t]{2}{*}{ CPT2 } & Forward & CATACAAGCTACATTTCGGGACC & NM_000098 \\
\hline & Reverse & AGCCCGGAGTGTCTTCAGAA & \\
\hline \multirow[t]{2}{*}{ OGDH } & Forward & GGCTTCCCAGACTGTTAAGAC & NM_001003941 \\
\hline & Reverse & GCAGAATAGCACCGAATCTGTTG & \\
\hline \multirow[t]{2}{*}{$\mathrm{CS}$} & Forward & TGCTTCCTCCACGAATTTGAAA & NM_004077 \\
\hline & Reverse & CCACCATACATCATGTCCACAG & \\
\hline \multirow[t]{2}{*}{ IDH2 } & Forward & CGCCACTATGCCGACAAAAG & NM_002168 \\
\hline & Reverse & ACTGCCAGATAATACGGGTCA & \\
\hline \multirow[t]{2}{*}{ IDH3a } & Forward & CCCGCGTGGATCTCTAAGG & NM_005530 \\
\hline & Reverse & AATTTCTGGGCCAATACCATCTC & \\
\hline \multirow[t]{2}{*}{$\mathrm{ACO} 2$} & Forward & CCCTACAGCCTACTGGTGACT & NM_001098 \\
\hline & Reverse & TGTACTCGTTGGGCTCAAAGT & \\
\hline \multirow[t]{2}{*}{ SDHA } & Forward & CAAACAGGAACCCGAGGTTTT & NM_004168 \\
\hline & Reverse & CAGCTTGGTAACACATGCTGTAT & \\
\hline \multirow[t]{2}{*}{ CYCS } & Forward & CTTTGGGCGGAAGACAGGTC & NM_018947 \\
\hline & Reverse & TTATTGGCGGCTGTGTAAGAG & \\
\hline \multirow[t]{2}{*}{$\operatorname{Cox} 4$} & Forward & CAGGGTATTTAGCCTAGTTGGC & NM_001861 \\
\hline & Reverse & GCCGATCCATATAAGCTGGGA & \\
\hline
\end{tabular}

${ }^{a} \mathrm{NCBI}$ accession nos. of genes (https://www.ncbi.nlm.nih.gov/nuccore). ACTB, $\beta$-actin; GLUT, glucose transporter; GLUT4, glucose transporter type 4; CPT, carnitine palmitoyl transferase; OGDH, oxoglutarate dehydrogenase; CS, citrate synthase; IDH, isocitrate dehydrogenase; $\mathrm{ACO} 2$, aconitate hydratase, mitochondrial; SDHA, succinate dehydrogenase complex flavoprotein subunit A; CYCS, cytochrome $c$; Cox4, CYCS oxidase IV.

$95^{\circ} \mathrm{C}$ for $2 \mathrm{~min}$, followed by 40 cycles of $95^{\circ} \mathrm{C}$ for $15 \mathrm{sec}$ and $56^{\circ} \mathrm{C}$ for $1 \mathrm{~min}$. Each analysis was performed in three to six replicates. The results are presented as the ratio of mtDNA relative to nuclear DNA.

Statistical analysis. Results were presented as mean \pm standard error of the mean of at least three independent experiments. The data were analysed with an unpaired Student's t-test using GraphPad Prism 5.0 (GraphPad Software, Inc., La Jolla, CA, USA). $\mathrm{P}<0.05$ was considered to indicate a statistically significant difference.

\section{Results}

Mutation of IDH1 exacerbates FA-induced apoptosis in HCT116 cells. Prior to the addition of FAs, the IDH1 (R132H) mutant HCT116 cells exhibited intrinsic vulnerability to the absence of glucose absence, which was consistent with a recent study (21). To investigate the roles of FA on cell viability, the parental and IDH1 mutant HCT116 cells were treated with serial concentrations of PA or OA. In the presence of $5.5 \mathrm{mM}$ glucose, lower concentrations $(50-200 \mu \mathrm{M})$ of PA or OA promoted the viability of parental and IDH1 mutant HCT116 cells, whereas a higher concentration $(400 \mu \mathrm{M})$ notably inhibited cell viability (Fig. 1A); however, in the absence of glucose, a high concentration $(400 \mu \mathrm{M})$ of $\mathrm{OA}(\mathrm{t}=5.751, \mathrm{P}<0.001)$ or $\mathrm{PA}$ $(\mathrm{t}=6.029, \mathrm{P}<0.001)$ significantly suppressed the viability of IDH1 mutant HCT116 cells compared with the parental cells, while lower concentrations (50 and $100 \mu \mathrm{M}$ ) of PA significantly increased cell viability (Fig. 1B). Furthermore, in the absence of glucose, the results of PI staining indicated that a high concentration $(400 \mu \mathrm{M})$ of PA or OA significantly stimulated the apoptosis of IDH1 mutant HCT116 cells compared with parental cells; however, in the presence of glucose, the percentage of apoptotic cells was markedly altered in IDH1 mutant HCT116 cells compared with the parental cells, when cultured with OA $(\mathrm{t}=5.749, \mathrm{P}<0.001)$ or $\mathrm{PA}(\mathrm{t}=6.030, \mathrm{P} \leq 0.001)$ (Fig. 1C and D). In summary, these results indicated that IDH1 mutant cells were more sensitive to FA-induced suppressed viability and apoptosis in the absence of glucose. 
A

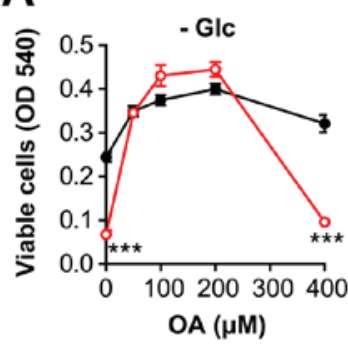

c
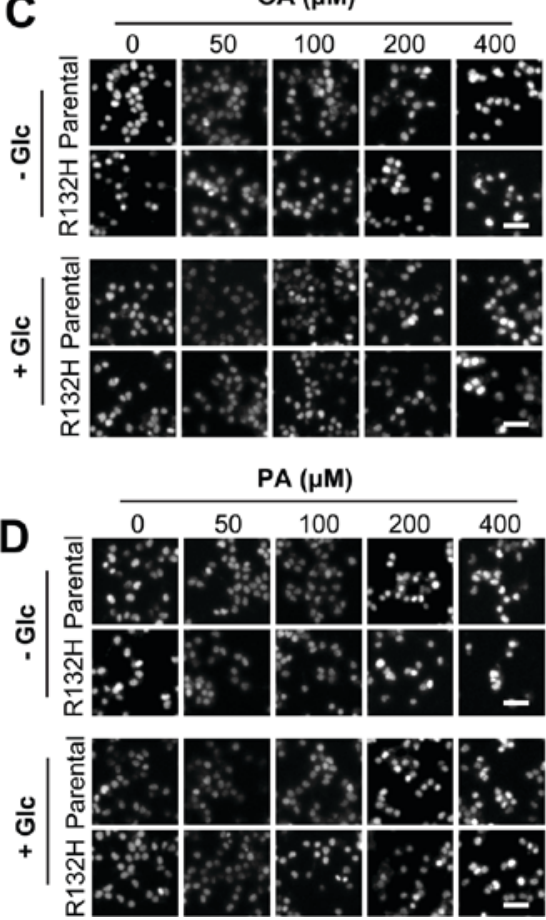

B
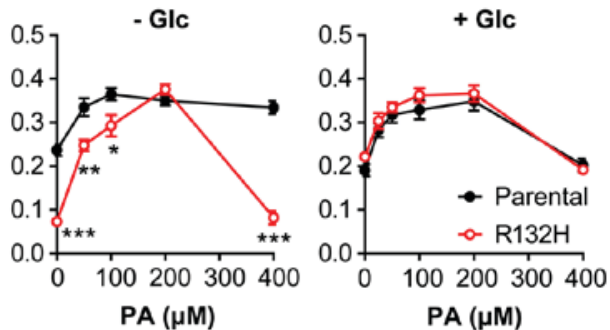
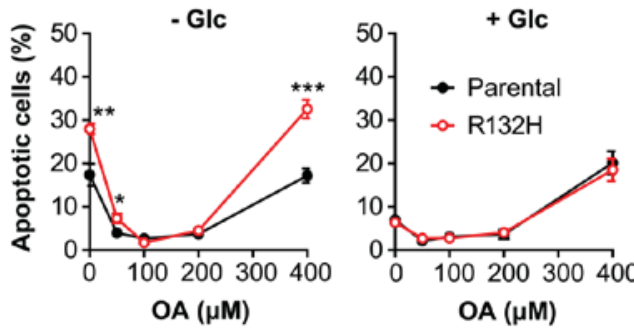

$\mathrm{OA}(\mu \mathrm{M})$
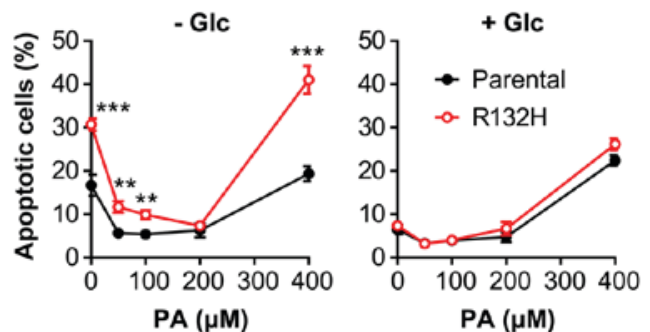

Figure 1. Mutation of IDH1 exacerbates the effects of fatty acids on the viability and apoptosis of HCT116 cells in the absence of glucose. Parental and IDH1 (R132H) mutant HCT116 cells were treated for $24 \mathrm{~h}$ with 0, 50, 100, 200 and $400 \mu \mathrm{M}$ (A) OA or (B) PA in the presence or absence of glucose, respectively. The number of viable cells was determined by an MTT assay. Percentages of apoptotic cells were determined by propidium iodide staining. Scale bar, $50 \mu \mathrm{m}$. (C) Cells treated with OA; (D) Cells treated with PA. The results are presented as the mean \pm standard error of the mean $(\mathrm{n}=6)$. ${ }^{*} \mathrm{P}<0.05,{ }^{* *} \mathrm{P}<0.01,{ }^{* * * *} \mathrm{P}<0.001$ vs. parental cells. IDH1, isocitrate dehydrogenase 1; OA, oleic acid; PA, palmitic acid; +Glc, presence of glucose; -Glc, absence of glucose.

Mutation of IDHI aggravates FA-induced oxidative stress in HCT116 cells. To investigate FA-induced suppressed viability and apoptosis, the intracellular ROS levels were determined in the parental and IDH1 mutant HCT116 cells treated with PA or OA. The results of DCFH-DA staining demonstrated that the absence of glucose significantly increased OA$(\mathrm{t}=3.479, \mathrm{P}<0.01)$ and $\mathrm{PA}(\mathrm{t}=3.900, \mathrm{P}<0.01)$-induced ROS production in IDH1 mutant HCT116 cells compared with parental cells (Fig. 2A-C). Additionally, the ROS levels in OA- or PA-treated IDH1 mutant HCT116 cells in the presence of glucose were notably increased compared with the parental cells (Fig. 2A-C).

Furthermore, the levels of MDA and 4-HNE, two end products of lipid peroxidation $(22,23)$, were significantly increased in IDH1 mutant HCT116 cells compared with the parental cells treated with $\mathrm{OA}(\mathrm{t}=2.470, \mathrm{P}<0.05$ and $\mathrm{t}=2.317, \mathrm{P}<0.05$, respectively) or $\mathrm{PA}(\mathrm{t}=2.910, \mathrm{P}<0.05$ and $\mathrm{t}=3.842, \mathrm{P}<0.01$, respectively) in the absence of glucose (Fig. 2D and E). Conversely, the addition of glucose markedly affected the levels of MDA and 4-HNE in parental and IDH1 mutant HCT116 cells (Fig. 2D and E). In summary, these results demonstrated that mutation of IDH1 promoted FA-induced production of ROS in HCT116 cells, in the absence of glucose.

Mutation of IDH1 inhibits FAO and increases glucose consumption. Excess intracellular FAs are primarily stored in LDs in the form of TG (24). In the present study, the results of Bodipy 493/503 staining demonstrated that there were significantly more LDs in IDH1 mutant HCT116 cells compared with in the parental cells following the treatment with $\mathrm{OA}(\mathrm{t}=3.005$, $\mathrm{P}<0.05)$ or $\mathrm{PA}(\mathrm{t}=2.927, \mathrm{P}<0.05)$ in the absence of glucose; however, the addition of glucose notably increased the number and size of LDs in parental and IDH1 mutant HCT116 cells (Fig. 3A-C). Quantitative analysis of intracellular TG content revealed similar results (Fig. 3D).

Furthermore, the oxidation of $\mathrm{OA}$ and PA was significantly reduced in IDH1 mutant HCT116 cells compared with parental cells (Fig. 3E; $\mathrm{t}=5.286, \mathrm{P}<0.01$; and $\mathrm{t}=3.013, \mathrm{P}<0.05$, respectively) in the absence of glucose at $8 \mathrm{~h}$; however, the addition of glucose suppressed the rate of FAO in parental and IDH1 mutant cells, but the difference between the groups was 
A
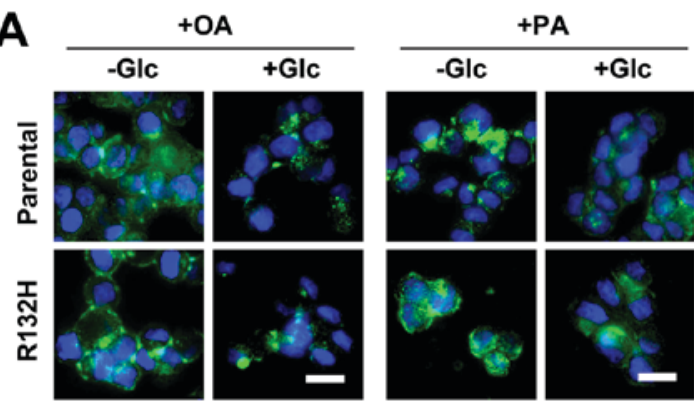

C
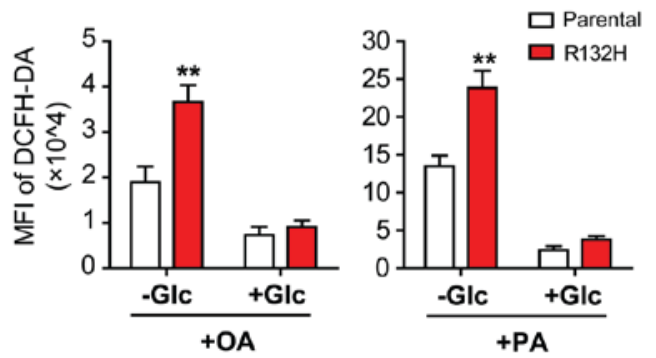

E

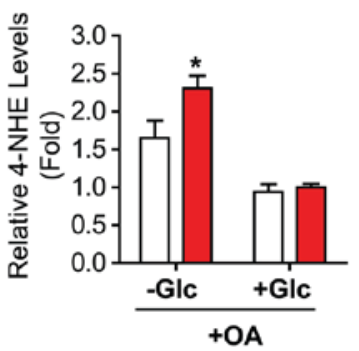

B

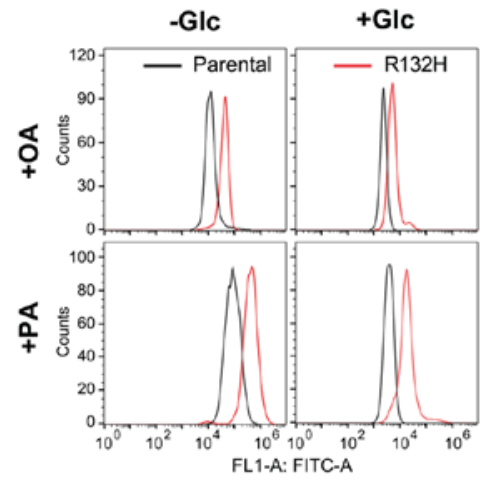

D

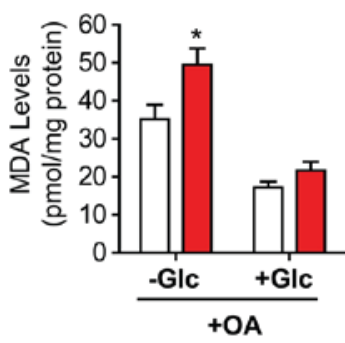

Figure 2. Mutation of IDH1 aggravates fatty acid-induced oxidative stress in HCT116 cells in the absence of glucose. Parental and IDH1 (R132H) mutant HCT116 cells were treated with $400 \mu \mathrm{M} \mathrm{OA}$ or PA for $24 \mathrm{~h}$ in the presence or absence of glucose. The intracellular reactive oxygen species levels were evaluated using (A) DCFH-DA staining and quantified using (B and C) flow cytometry. Scale bar=20 $\mu \mathrm{m}$. The expression levels of (D) MDA and (E) 4-HNE were determined. The results were presented as the mean \pm standard error of the mean $(\mathrm{n}=6) .{ }^{*} \mathrm{P}<0.05,{ }^{* *} \mathrm{P}<0.01$ vs. parental cells. IDH1, isocitrate dehydrogenase 1 ; OA, oleic acid; PA, palmitic acid; DCFH-DA, dichloro-dihydro-fluorescein diacetate; +Glc, presence of glucose; -Glc, absence of glucose; MDA, malondialdehyde; 4-HNE, 4-hydroxy-2-nonenal; MFI, mean fluorescence intensity.

not significant (Fig. 3E). In addition, the results demonstrated that the levels of glucose in culture media decreased more rapidly in IDH1 mutant cells compared with the parental cells, when treated with $\mathrm{OA}(\mathrm{t}=4.714, \mathrm{P}<0.01$ at $4 \mathrm{~h})$ or $\mathrm{PA}(\mathrm{t}=3.768$, $\mathrm{P}<0.01$ at $4 \mathrm{~h})($ Fig. $3 \mathrm{~F})$, which indicated that IDH1 mutant HCT116 cells consumed more glucose than the parental cells. In summary, these results suggested that mutation of IDH1 reduced the rate of FAO, but promoted glucose consumption in HCT116 cells.

Mutation of IDHI disrupts the mitochondrial respiratory chain. To confirm the elevated ROS production and reduced FAO rate in IDH1 mutant HCT116 cells, mitochondrial function was further analyzed. The results of MitoTracker staining and mitochondrial DNA quantification demonstrated that the quantity of mitochondria in IDH1 mutant HCT116 cells was similar to that of the parental cells (Fig. 4A). Furthermore, the results of RT-qPCR indicated that the mRNA expression levels of GLUT1 were significantly increased $(\mathrm{t}=3.356, \mathrm{P}<0.01$; Fig. 4B) compared with parental cells; however, that of CPT1, CYCS and Cox 4 were significantly reduced in IDH1 mutant HCT116 cells $(\mathrm{t}=2.537,2.567$ and 5.039; $\mathrm{P}<0.05,0.05$ and 0.01 , respectively). The mRNA expression levels of GLUT4, CPT2, oxoglutarate dehydrogenase, citrate synthase, IDH2, IDH3a, aconitase 2 and succinate dehydrogenase complex flavoprotein subunit A were notably altered in IDH1 mutant HCT116 cells compared with in the parental cells. Immunoblotting demonstrated that the protein expression levels of GLUT1 were markedly upregulated, but the protein levels of CPT1, CYCS and Cox4 were notably downregulated in IDH1 mutant HCT116 cells treated with PA or OA in the presence or absence of glucose compared with in the parental cells, which were consistent with the results of RT-qPCR (Fig. 4C); reduced expression of CYCS and Cox4 in IDH1 mutant HCT116 cells indicated that the mutation of IDH1 impaired the mitochondrial respiratory chain. 
A

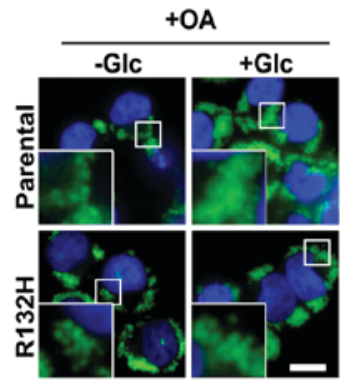

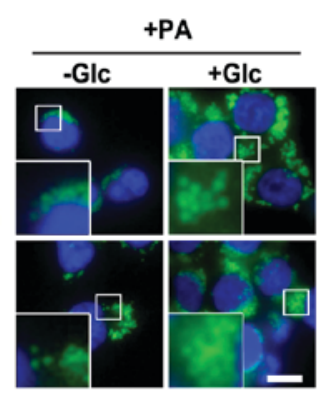

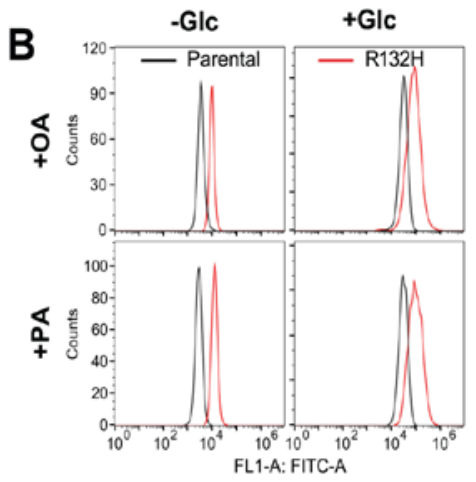

C
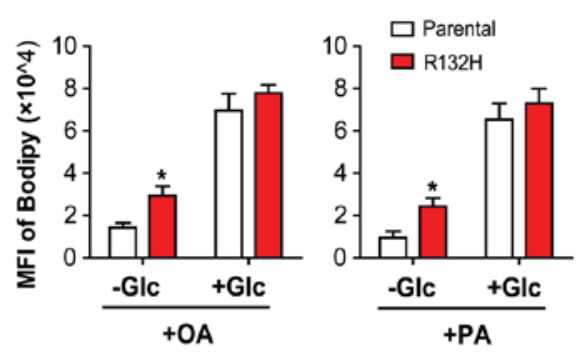

E

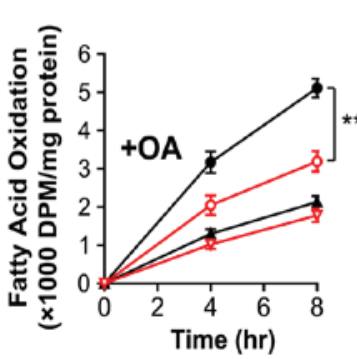

- Parental wio Glc - R132H w/o Glc 3.07 — Parental + Glc $2.5-\rightarrow \mathrm{R} 132 \mathrm{H}+\mathrm{Glc}$

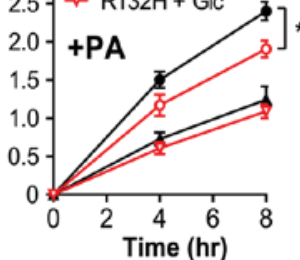

$2.0-1$.
D
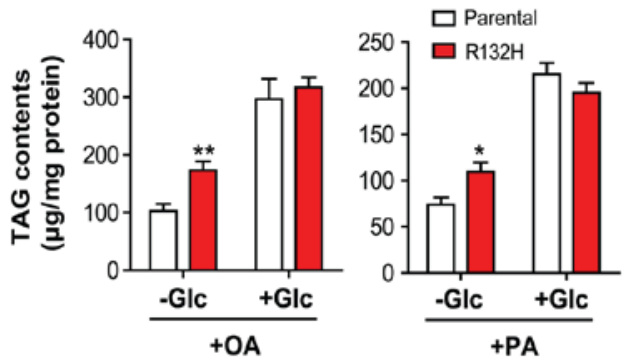

$\mathbf{F}$

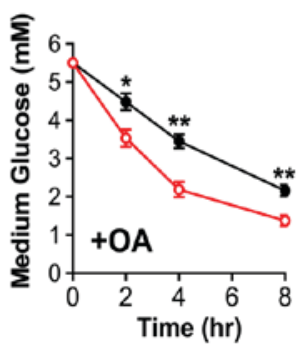

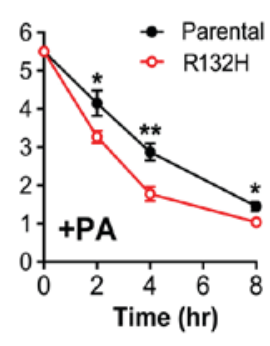

Figure 3. Mutation of IDH1 inhibits FAO and increases glucose consumption in HCT116 cells. Parental and IDH1 (R132H) mutant HCT116 cells were treated with $400 \mu \mathrm{M}$ OA or PA for $24 \mathrm{~h}$, in the presence or absence of glucose. The intracellular lipid droplets were stained with (A) Bodipy $493 / 503$ and quantified using (B and C) flow cytometry. Scale bar=20 $\mu \mathrm{m}$. (D) Total lipids were extracted and the intracellular TG levels were determined. (E) Parental and IDH1 (R132H) mutant HCT116 cells were cultured with ${ }^{3} \mathrm{H}$-labelled OA and PA in the presence and absence of glucose. The rate of FAO was presented as the production of radioactive water or water-soluble metabolites following incubation for 4 and $8 \mathrm{~h}$. (F) Parental and IDH1 (R132H) mutant HCT116 cells were treated with $400 \mu \mathrm{M} \mathrm{OA}$ or PA for $24 \mathrm{~h}$ in the presence of glucose, and the glucose levels in the media were determined. The results were presented as the mean \pm standard error of the mean $(n=4) .{ }^{*} \mathrm{P}<0.05,{ }^{* *} \mathrm{P}<0.01$ vs. parental cells. IDH1, isocitrate dehydrogenase 1; OA, oleic acid; PA, palmitic acid; +Glc, presence of glucose; -Glc, absence of glucose; FAO, fatty acid oxidation; TG, triglyceride; MFI, mean fluorescence intensity; DPM, disintegrations per minute.

\section{Discussion}

ROS in cancer cells are frequently upregulated, and elevated ROS is pro-tumorigenic, consequently resulting in the activation of pro-survival signaling pathways, increased glucose metabolism, adaptation to hypoxia and the generation of oncogenic mutations (25); however, toxic levels of ROS in cancer cells are also anti-tumorigenic by increasing oxidative stress and inducing tumour cell death $(26,27)$. In clinical practice, numerous chemotherapy drugs increase the production of ROS to toxic levels and exhaust the capacity of the antioxidant system to induce growth arrest and cell death (28); thus, eliminating or elevating ROS production may be effective therapeutic strategies for the treatment of cancer.

IDH mutations have been reported in glioma, AML, enchondroma and chondrosarcoma (7-10). Wild type IDH1/2 catalysed the conversion of isocitrate into $\alpha-K G$ with the reduction of $\mathrm{NADP}^{+}$and functioned in protecting cells from oxidative stress by regulating the intracellular $\mathrm{NADP}^{+} / \mathrm{NADPH}$ ratio (11). Wild type IDH1 reduced ROS following treatment with lipopolysaccharide in vitro (29), and IDH1-null hepatocytes also exhibited upregulated intracellular ROS; however, the oncogenic IDH mutations served a novel function to catalyse the reduction of $\alpha-\mathrm{KG}$ to $2-\mathrm{HG}$ by oxidizing NADPH (13). A recent study indicated that 2-HG inhibited ATP synthase and mechanistic target of rapamycin signalling in glioblastoma cells, consequently inducing growth arrest and tumour cell death in the absence of glucose (21). Furthermore, it was demonstrated that ROS generation was elevated in IDH1 mutant cells, and the potential mechanism was due to decreased NADPH, which may suppress the conversion of oxidized glutathione (GSH) disulfide into GSH (30); however, the effects of IDH1 mutation on lipid metabolism and mitochondrial functions remain unknown.

A recent study demonstrated that cancer cells primarily cultured under serum-free conditions exhibited the ability to oxidize FA, in order to maintain respiratory and proliferative activity (31). OA (C18:1) and PA (C16:0) are the most 

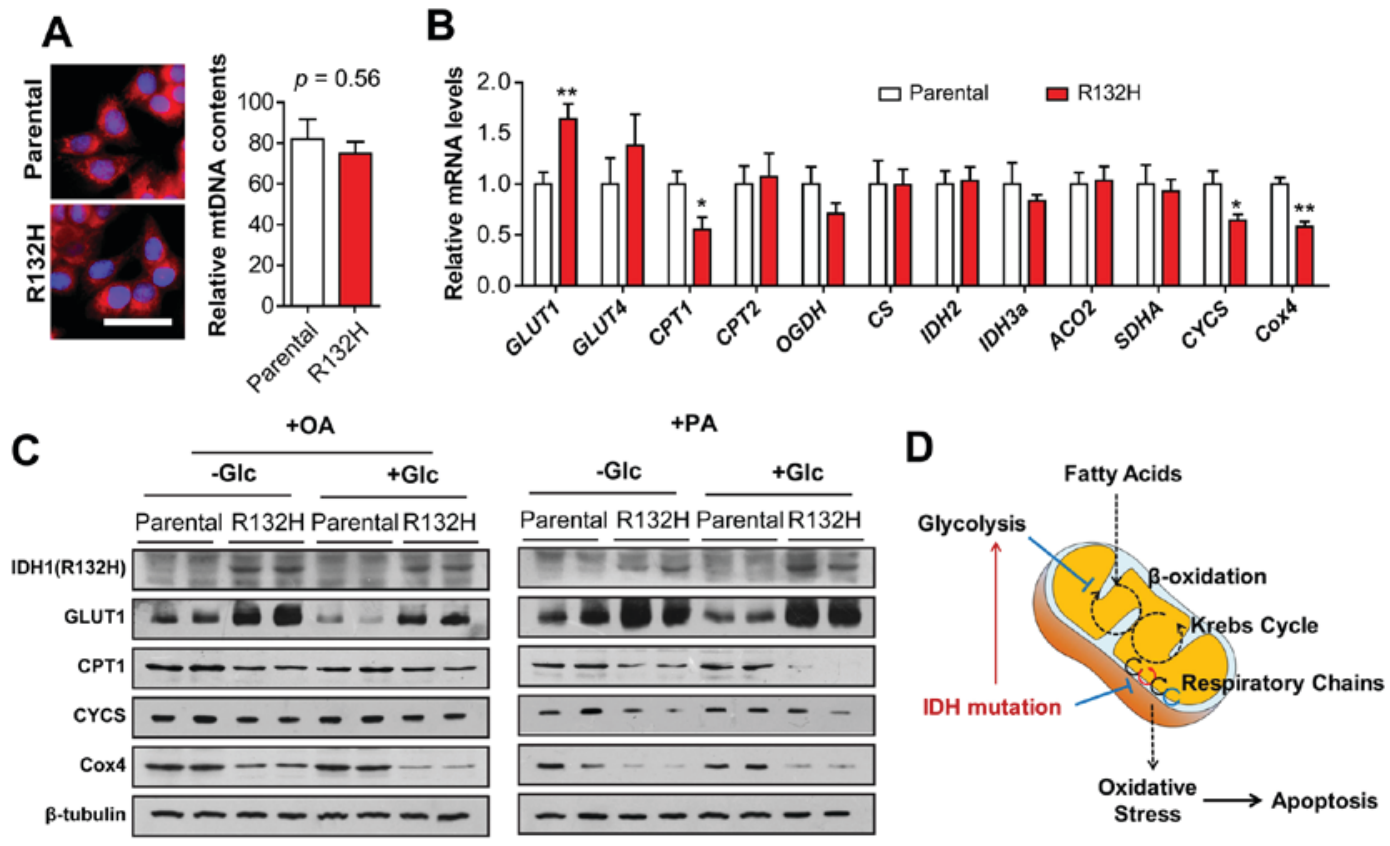

Figure 4. Mutation of IDH1 impairs the respiratory chain in mitochondria. (A) Quantity of mitochondria and mtDNA in parental and IDH1 (R132H) mutant HCT116 cells were determined using MitoTracker staining and mtDNA quantification. Scale bar=20 $\mu \mathrm{m}$. (B) mRNA levels of GLUT1, GLUT4, CPT1, CPT2, OGDH, CS, IDH2, IDH3a, ACO2, SDHA, CYCS and Cox4 in parental and IDH1 (R132H) mutant HCT116 cells were determined using reverse transcription-quantitative polymerase chain reaction. The results were presented as the mean \pm standard error of the mean $(\mathrm{n}=4)$. ${ }^{*} \mathrm{P}<0.05$, ${ }^{* * *} \mathrm{P}<0.01 \mathrm{vs}$. parental cells. (C) Parental and IDH1 (R132H) mutant HCT116 cells were treated with PA or OA for $24 \mathrm{~h}$ in the presence or absence of glucose. The protein levels of GLUT1, CPT1, CYCS, and Cox4 were determined using western blotting analysis. (D) Schematic diagram revealed that mutation of IDH1 aggravates fatty acid-induced oxidative stress in HCT116 cells. IDH, isocitrate dehydrogenase; OA, oleic acid; PA, palmitic acid; +Glc, presence of glucose; -Glc, absence of glucose; GLUT, glucose transporter; CPT, carnitine palmitoyl transferase; OGDH, oxoglutarate dehydrogenase; CS, citrate synthase; ACO2, aconitase 2; SDHA, succinate dehydrogenase complex flavoprotein subunit A; CYCS, cytochrome $c$; Cox4, CYCS oxidase IV; mtDNA, mitochondrial DNA.

abundant dietary and plasma FAs (32). As a saturated FA, PA serves prominent roles in perturbing the lipid composition in membranes, resulting in endoplasmic reticulum stress and mitochondrial dysfunction (33-35). In the present study, it was determined that lower concentrations $(50-200 \mu \mathrm{M})$ of PA or OA promoted the viability of parental and IDH1 mutant HCT116 cells in the absence of glucose; however, a higher concentration of PA or OA $(400 \mu \mathrm{M})$ induced the apoptosis and suppressed the viability of IDH1 mutant cells by increasing ROS production and lipid peroxidation in the absence of glucose. In addition, the results of the present study indicated that mutation of IDH1 inhibited FAO in HCT116 cells, resulting in increased TG accumulation in the absence of glucose.

Among the mitochondrial metabolic pathways, FAO is of particular interest as the inhibition of FAO may be a potential target for reducing tumor growth (36). Regarding metabolic stress, the production of FAO-derived cytosolic NADPH by cancer cells may be key to counteract oxidative stress. In the present study, decreased $\beta$-oxidation of FA and the activity of mutant IDH may have also decreased the levels of reducing equivalents, aggravating oxidative stress in IDH mutant cells. Furthermore, during the production of ATP via oxidative phosphorylation, mitochondria are the primary intracellular producers of ROS and $\sim 0.1-2 \%$ of the $\mathrm{O}_{2}$ consumed by the mitochondria is used to produce $\mathrm{O}_{2}(37,38)$, particularly at the levels of complexes I and III in the mitochondrial electron transport chain (ETC). In the present study, mutation of
IDH1 downregulated the expression of CYCS and Cox4 in the mitochondrial respiratory chain. As a component of the mitochondrial ETC, CYCS serves crucial roles in transferring electrons between complexes III (coenzyme Q-CYCS reductase) and IV (CYCS oxidase), and the reduced levels of CYCS and Cox4 may also contribute to the elevated mitochondrial ROS production in IDH1 mutant HCT116 cells (Fig. 4D). Additionally, peroxisomes are sources of cytosolic $\mathrm{H}_{2} \mathrm{O}_{2}$ under physiological conditions (39). Furthermore, endoplasmic reticular monooxygenases, such as cytochrome P450, also contribute to increased cellular ROS levels, which promote lipid peroxidation, altered calcium homeostasis, mitochondrial dysfunction and cell apoptosis $(40,41)$; however, the effects of IDH1 mutations on other cellular sources of ROS require further investigation.

Accumulating oxidative damage may affect the efficiency of mitochondria and further promote the ROS generation. In order to determine the association between mitochondrial dysfunction and oxidative stress in IDH mutant cells, the essential roles of mutant IDH in regulating gene transcription were investigated in the present study. CPT1 is an transporter associated with the outer mitochondrial membrane (42) and is required to transport long-chain FAs into the mitochondrial matrix (43). GLUT1 facilitates the transport of glucose across the plasma membranes of mammalian cells $(36,44)$. In the present study, it was determined that mutation of IDH1 downregulated the expression levels of CPT1, but upregulated those of GLUT1, which coincided with reduced FAO and increased 
glycolysis in IDH1 mutant HCT116 cells. The aberrant expression of GLUT1 and CPT1 may be attributed to the alterations of epigenetics and hypoxia inducible factor- 1 in cancer types with IDH mutation; however, their roles in tumorigenesis require further investigation (45).

IDH mutations may affect cellular metabolism, epigenetics and other biochemical functions (13), but their roles in lipid metabolism remain unknown. In the present study, the data indicated that mutation of IDH1 aggravated FA-induced oxidative stress in HCT116 cells by reducing FAO and disrupting mitochondrial function. These results are not only important for understanding the roles of IDH mutation in tumour metabolic reprogramming, but also provide novel insight into potential therapeutic strategies for the treatment of cancers with IDH mutation.

\section{Acknowledgements}

Not applicable.

\section{Funding}

The present study was funded by the National Natural Science Foundation of China (grant nos. 81572471 and 81370958), the Natural Science Foundation of Shanxi Province China (grant no. 2016JM8100), the Booster Program of Xijing Hospital (grant no. XJZT15ZL03), the Key Laboratory free exploration project (grant no. CBSKL2015Z11) and the Youth Science Foundation of Xi'an Medical College (grant no. 2015QN01).

\section{Availability of data and materials}

All data generated or analyzed during this study are included in this published article.

\section{Authors' contributions}

SL performed the administration and cell culture and revised the figures. CS and YG performed the PI staining, MitoTracker staining, DCFH-DA staining and Bodipy staining. XG, YZ and YY performed the determination of FAO. FZ and PH performed the western blot analysis and RT-qPCR. WL, KC and JZ performed the determination of MDA and 4-HNE and glucose consumption analysis. JY and $\mathrm{ZW}$ contributed to the experimental design and drafted the manuscript. All authors read and approved the final manuscript.

\section{Ethics approval and consent to participate}

Not applicable.

\section{Patient consent for publication}

Not applicable.

\section{Competing interests}

The authors declare that they have no competing interests.

\section{References}

1. Hanahan D and Weinberg RA: Hallmarks of cancer: The next generation. Cell 144: 646-674, 2011.

2. Huang $\mathrm{C}$ and Freter C: Lipid metabolism, apoptosis and cancer therapy. Int J Mol Sci 16: 924-949, 2015.

3. Currie E, Schulze A, Zechner R, Walther TC and Farese RV Jr: Cellular fatty acid metabolism and cancer. Cell Metab 18: 153-161, 2013.

4. Carracedo A, Cantley LC and Pandolfi PP: Cancer metabolism: Fatty acid oxidation in the limelight. Nat Rev Cancer 13: 227-232, 2013.

5. Tafani M, Sansone L, Limana F, Arcangeli T, De Santis E, Polese M, Fini M and Russo MA: The interplay of reactive oxygen species, hypoxia, inflammation, and sirtuins in cancer initiation and progression. Oxid Med Cell Longev 2016: 3907147, 2016.

6. Kim J, Kim J and Bae JS: ROS homeostasis and metabolism: A critical liaison for cancer therapy. Exp Mol Med 48: e269, 2016.

7. Reitman ZJ and Yan H: Isocitrate dehydrogenase 1 and 2 mutations in cancer: Alterations at a crossroads of cellular metabolism. J Natl Cancer Inst 102: 932-941, 2010.

8. Hirata M, Sasaki M, Cairns RA, Inoue S, Puviindran V, Li WY, Snow BE, Jones LD, Wei Q, Sato S, et al: Mutant IDH is sufficient to initiate enchondromatosis in mice. Proc Natl Acad Sci USA 112: 2829-2834, 2015.

9. Sasaki M, Knobbe CB, Munger JC, Lind EF, Brenner D, Brüstle A, Harris IS, Holmes R, Wakeham A, Haight J, et al: IDH1(R132H) mutation increases murine haematopoietic progenitors and alters epigenetics. Nature 488: 656-659, 2012.

10. Cairns RA, Iqbal J, Lemonnier F, Kucuk C, de Leval L, Jais JP, Parrens M, Martin A, Xerri L, Brousset P, et al: IDH2 mutations are frequent in angioimmunoblastic T-cell lymphoma. Blood 119: 1901-1903, 2012

11. Xu X, Zhao J, Xu Z, Peng B, Huang Q, Arnold E and Ding J: Structures of human cytosolic NADP-dependent isocitrate dehydrogenase reveal a novel self-regulatory mechanism of activity. J Biol Chem 279: 33946-33957, 2004.

12. Ye J, Gu Y, Zhang F, Zhao Y, Yuan Y, Hao Z, Sheng Y, Li WY, Wakeham A, Cairns RA and Mak TW: IDH1 deficiency attenuates gluconeogenesis in mouse liver by impairing amino acid utilization. Proc Natl Acad Sci USA 114: 292-297, 2017.

13. Cohen AL, Holmen SL and Colman H: IDH1 and IDH2 mutations in gliomas. Curr Neurol Neurosci Rep 13: 345, 2013.

14. Ma S, Jiang B, Deng W, Gu ZK, Wu FZ, Li T, Xia Y, Yang H, Ye D, Xiong Y and Guan KL: D-2-hydroxyglutarate is essential for maintaining oncogenic property of mutant IDH-containing cancer cells but dispensable for cell growth. Oncotarget 6: 8606-8620, 2015.

15. Lazarow PB: The role of peroxisomes in mammalian cellular metabolism. J Inherit Metab Dis 10 (Suppl 1): S11-S22, 1987.

16. Shechter I, Dai P, Huo L and Guan G: IDH1 gene transcription is sterol regulated and activated by SREBP-1a and SREBP-2 in human hepatoma HepG2 cells: Evidence that IDH1 may regulate lipogenesis in hepatic cells. J Lipid Res 44: 2169-2180, 2003.

17. Bogdanovic E: IDH1, lipid metabolism and cancer: Shedding new light on old ideas. Biochim Biophys Acta 1850: 1781-1785, 2015.

18. Li F, He X, Ye D, Lin Y, Yu H, Yao C, Huang L, Zhang J, Wang F, $\mathrm{Xu}$ S, et al: $\mathrm{NADP}(+)-\mathrm{IDH}$ mutations promote hypersuccinylation that impairs mitochondria respiration and induces apoptosis resistance. Mol Cell 60: 661-675, 2015.

19. Jin G, Reitman ZJ, Duncan CG, Spasojevic I, Gooden DM, Rasheed BA, Yang R, Lopez GY, He Y, McLendon RE, et al: Disruption of wild-type IDH1 suppresses D-2-hydroxyglutarate production in IDH1-mutated gliomas. Cancer Res 73: 496-501, 2013.

20. Livak KJ and Schmittgen TD: Analysis of relative gene expression data using real-time quantitative PCR and the 2(-Delta Delta C(T)) method. Methods 25: 402-408, 2001.

21. Fu X, Chin RM, Vergnes L, Hwang H, Deng G, Xing Y, Pai MY, Li S, Ta L, Fazlollahi F, et al: 2-hydroxyglutarate inhibits ATP synthase and mTOR signaling. Cell Metab 22: 508-515, 2015.

22. Gutiérrez AM, Reboredo GR and Catalá A: Fatty acid profiles and lipid peroxidation of microsomes and mitochondria from liver, heart and brain of Cairina moschata. Int J Biochem Cell Biol 34: 605-612, 2002.

23. Rohn TT, Nelson LK, Waeg G and Quinn MT: U-101033E (2,4-diaminopyrrolopyrimidine), a potent inhibitor of membrane lipid peroxidation as assessed by the production of 4-hydroxynonenal, malondialdehyde, and 4-hydroxynonenal-protein adducts. Biochem Pharmacol 56: 1371-1379, 1998. 
24. Nguyen P, Leray V, Diez M, Serisier S, Le Bloc'h J, Siliart B and Dumon H: Liver lipid metabolism. J Anim Physiol Anim Nutr (Berl) 92: 272-283, 2008.

25. Sabharwal SS and Schumacker PT: Mitochondrial ROS in cancer: Initiators, amplifiers or an Achilles' heel? Nat Rev Cancer 14: 709-721, 2014.

26. Glasauer A and Chandel NS: Targeting antioxidants for cancer therapy. Biochem Pharmacol 92: 90-101, 2014

27. Wu MS, Lien GS, Shen SC, Yang LY and Chen YC: $\mathrm{N}$-acetyl-L-cysteine enhances fisetin-induced cytotoxicity via induction of ROS-independent apoptosis in human colonic cancer cells. Molecular Carcinogenesis 53 (Suppl 1): E119-E129, 2014.

28. Pelicano H, Carney D and Huang P: ROS stress in cancer cells and therapeutic implications. Drug Resist Updat 7: 97-110, 2004

29. Itsumi M, Inoue S, Elia AJ, Murakami K, Sasaki M, Lind EF, Brenner D, Harris IS, Chio II, Afzal S, et al: Idh1 protects murine hepatocytes from endotoxin-induced oxidative stress by regulating the intracellular NADP(+)/NADPH ratio. Cell Death Differ 22: 1837-1845, 2015.

30. Shi J, Zuo H, Ni L, Xia L, Zhao L, Gong M, Nie D, Gong P, Cui D, Shi W and Chen J: An IDH1 mutation inhibits growth of glioma cells via GSH depletion and ROS generation. Neurol Sci 35: 839-845, 2014

31. Lin H, Patel S, Affleck VS, Wilson I, Turnbull DM, Joshi AR, Maxwell R and Stoll EA: Fatty acid oxidation is required for the respiration and proliferation of malignant glioma cells. Neuro Oncol 19: 43-54, 2017.

32. Staiger H, Staiger K, Stefan N, Wahl HG, Machicao F, Kellerer M and Häring HU: Palmitate-induced interleukin-6 expression in human coronary artery endothelial cells. Diabetes 53: 3209-3216, 2004.

33. Palomer X,Pizarro-Delgado J,Barroso Eand Vázquez-Carrera M: Palmitic and oleic acid: The Yin and Yang of fatty acids in type 2 diabetes mellitus. Trends Endocrinol Metab 29: 178-190, 2018.

34. Leamy AK, Egnatchik RA, Shiota M, Ivanova PT, Myers DS, Brown HA and Young JD: Enhanced synthesis of saturated phospholipids is associated with ER stress and lipotoxicity in palmitate treated hepatic cells. J Lipid Res 55: 1478-1488, 2014.

35. Moravcová A, Červinková Z, Kučera O, Mezera V, Rychtrmoc D and Lotková $\mathrm{H}$ : The effect of oleic and palmitic acid on induction of steatosis and cytotoxicity on rat hepatocytes in primary culture. Physiol Res 64 (Suppl 5): S627-S636, 2015.
36. Tirado-Vélez JM, Joumady I, Sáez-Benito A, Cózar-Castellano I and Perdomo G: Inhibition of fatty acid metabolism reduces human myeloma cells proliferation. PLoS One 7: e46484, 2012.

37. Handy DE and Loscalzo J: Redox regulation of mitochondrial function. Antioxid Redox Signal 16: 1323-1367, 2012.

38. Quinlan CL, Treberg JR, Perevoshchikova IV, Orr AL and Brand MD: Native rates of superoxide production from multiple sites in isolated mitochondria measured using endogenous reporters. Free Radic Biol Med 53: 1807-1817, 2012.

39. Fritz R, Bol J, Hebling U, Angermüller S, Völkl A, Fahimi HD and Mueller S: Compartment-dependent management of $\mathrm{H}(2)$ $\mathrm{O}(2)$ by peroxisomes. Free Radic Biol Med 42: 1119-1129, 2007.

40. Zangar RC, Davydov DR and Verma S: Mechanisms that regulate production of reactive oxygen species by cytochrome P450. Toxicol Appl Pharmacol 199: 316-331, 2004.

41. Caro AA and Cederbaum AI: Role of cytochrome P450 in phospholipase A2- and arachidonic acid-mediated cytotoxicity. Free Radic Biol Med 40: 364-375, 2006.

42. McGarry JD and Brown NF: The mitochondrial carnitine palmitoyltransferase system. From concept to molecular analysis. Eur J Biochem 244: 1-14, 1997.

43. Palomer X, Salvadó L, Barroso E and Vázquez-Carrera M: An overview of the crosstalk between inflammatory processes and metabolic dysregulation during diabetic cardiomyopathy. Int J Cardiol 168: 3160-3172, 2013.

44. Olson AL and Pessin JE: Structure, function, and regulation of the mammalian facilitative glucose transporter gene family. Annu Rev Nutr 16: 235-256, 1996.

45. Young RM and Simon MC: Untuning the tumor metabolic machine: HIFa: Pro-and anti-tumorigenic? Nat Med 18: 1024-1025, 2012. 